\title{
Operationalisierung von Vertrauen in Journalismus
}

\author{
Jörg Matthes / Matthias Kohring
}

Thema des Beitrags ist die Operationalisierung von Vertrauen in medial vermittelte journalistische Kommunikation, kurz: Vertrauen in Journalismus. Zunächst erfolgt ein Überblick zur bisherigen Konzeptionalisierung und Operationalisierung von Vertrauen in Journalismus. Das kritische Fazit: Es liegt bislang keine theoretisch und methodisch fundierte Skala zur Erfassung von Vertrauen in Journalismus vor. Darüber hinaus sind Operationalisierungen von Vertrauen in Journalismus nicht konstruktvalidiert. Das Ziel des vorliegenden Beitrags ist es, an Hand eines theoretisch abgeleiteten mebrdimensionalen Modells eine Skala zur Erfassung von Vertrauen in Journalismus zu entwickeln und empirisch zu testen. Das zentrale Ergebnis einer Befragung ist die Bestätigung der vier postulierten Faktoren "Vertrauen in Themenselektivität", "Vertrauen in Faktenselektivität", "Vertranen in die Richtigkeit von Beschreibungen" (Glaubwürdigkeit) und "Vertrauen in Bewertungen" mittels einer konfirmatorischen Faktorenanalyse. Zudem kann im Rabmen einer ersten Konstruktvalidierung gezeigt werden, dass die Persönlichkeitseigenschaft „Vertrauensfähigkeit" ein signifikanter Prädiktor von Vertrauen in Journalismus ist.

Keywords: Vertrauen in Journalismus, Glaubwürdigkeit, Skalenentwicklung, konfirmatorische Faktorenanalyse, Konstruktvalidierung, Vertrauensfähigkeit

\section{Einleitung}

Seit den neunziger Jahren trifft der Vertrauensbegriff in den Sozialwissenschaften auf ein wiedererwachtes Interesse (vgl. Giddens ${ }^{2} 1997$; Earle/Cvetkovich 1995; Misztal 1996; Sztompka 1999; Hartmann/Offe 2001; Hardin 2002). Vertrauen gilt als eine wichtige Basis sozialer Ordnung bzw. als Grundlage des sozialen Zusammenhalts und somit als ein Schlüsselbegriff für das Funktionieren der modernen Gesellschaft mit ihrer Zukunftsoffenheit und Betonung riskanten Entscheidens. Allerdings hat die behauptete Relevanz des Begriffs methodologisch kaum Konsequenzen. Die empirische Beobachtung von Vertrauensprozessen erfolgt in der Regel als eine direkte und eindimensionale Abfrage des Vertrauens in bestimmte Akteure, wobei sehr oft nicht klar ist, was eigentlich erfasst wird (vgl. z. B. Chanley/Rudolph/Rahn 2000).

Dies gilt auch für die Kommunikations- und Medienwissenschaft, die bislang den Begriff der Glaubwürdigkeit präferiert hat. Theorien des Vertrauens haben hier noch so gut wie keinen Niederschlag gefunden (vgl. allerdings Bentele 1994b; 1998). Dies ist umso erstaunlicher, als Journalismus ohne ein bestimmtes Maß an Vertrauen, das ihm seine Publika Tag für Tag entgegenbringen, seine gesellschaftliche Orientierungsfunktion nicht erfüllen könnte: Vertrauen kann nachgerade als entscheidende Schlüsselvariable für „Medienwirkungen“ bezeichnet werden. Daher sind die möglicherweise vielfältigen Dimensionen, auf denen das Publikum seine Bewertungen journalistischer Vertrauenswürdigkeit trifft, von hohem theoretischen und praktischen Interesse. Wenn es nämlich gelingt, Vertrauen in Journalismus differenzierter als über ein globales und unspezifisches Glaubwürdigkeitsurteil zu erfassen, beinhaltet dies auch die Möglichkeit, spezifische Vertrauensprobleme journalistischer Publika zu identifizieren und hierfür konkrete Verbesserungsvorschläge zu unterbreiten. 
Nachdem an anderer Stelle schon eine ausführliche theoretische Diskussion des Forschungsstandes erfolgte (vgl. Kohring 2001; 2002a), liegt der Fokus dieses Beitrages auf der Operationalisierung von Vertrauen in medial vermittelte journalistische Kommunikation, im Folgenden kurz „Vertrauen in Journalismus“ genannt. ${ }^{1}$ Hierzu werden zunächst bisherige Operationalisierungen von Vertrauen in Journalismus kritisch diskutiert. Zwei Aspekte werden als Schwachpunkte der Forschung identifiziert: Zum Ersten existiert kein theoretisch und methodisch fundiertes Messinstrument, das die Analyse von Vertrauen in Journalismus ermöglicht. Zum Zweiten wurde Vertrauen in Journalismus noch keiner Konstruktvalidierung unterzogen (2). Im Folgenden geht es daher um die Konstruktion und Validierung einer Skala zur Messung von Vertrauen in Journalismus. Ausgangspunkt hierfür ist ein theoretisch abgeleitetes Vierfaktorenmodell, das u. a. auch den in der Kommunikations- und Medienwissenschaft alternativ zu Vertrauen verwendeten Begriff der Glaubwürdigkeit einschließt (3). Im Anschluss wird das methodische Vorgehen zur Operationalisierung und empirischen Überprüfung dieses Konstrukts vorgestellt (4). Die Ergebnisse einer standardisierten Umfrage bestätigen das vorgeschlagene Faktorenmodell (5). Der Beitrag schließt mit einer kritischen Diskussion und Einordnung der vorgeschlagenen Operationalisierung von Vertrauen in Journalismus (6).

\section{Forschungsstand}

Die Forschung zu Vertrauen in Journalismus ist fast ausschließlich unter dem Begriff der Medienglaubwürdigkeit bekannt geworden (zum Verhältnis der beiden Begriffe vgl. 3). Folgende drei Ansätze lassen sich unterscheiden (vgl. Kohring 2001):

(1) der Roper-Ansatz zur vergleichenden Medienglaubwürdigkeit

(2) der faktorenanalytische Ansatz der Glaubwürdigkeitsforschung

(3) der objektivitätsorientierte Ansatz

Der folgende Überblick gilt vor allem den Operationalisierungen des Konstrukts Glaubwürdigkeit resp. Vertrauen.

Die vergleichende Medienglaubwürdigkeitsforschung findet ihren Ursprung in den Untersuchungen von Elmo Roper zur Glaubwürdigkeit von Radio, Fernsehen, Zeitschrift und Zeitung. Ab 1959 wurde dem amerikanischen Publikum die so genannte Roper-Frage im Abstand von zwei Jahren gestellt (in Deutschland wurde die Frage in die Langzeitstudie Massenkommunikation übernommen; vgl. Berg/Kiefer 1996; vgl. schon Drescher 1969): "If you got conflicting or different reports of the same story from radio, television, the magazines and the newspapers, which of the four versions would you be most inclined to believe - the one on radio or television or magazines or newspapers?" (Roper 1985: 5)

Diese Frage wurde in zahlreichen weiteren Umfragen eingesetzt (vgl. z. B. Westley/Severin 1964; Greenberg/Razinsky 1966; Drescher 1969; Shaw 1973; Mulder 1980). Während die Tageszeitung in den USA bis 1961 noch als glaubwürdigstes Informationsmedium galt, zeigen spätere Roper-Daten einen angeblichen deutlichen „Glaubwürdigkeitsvorsprung“ des Fernsehens.

Die Roper-Frage hat von vielen Seiten eine mitunter vehemente Kritik erfahren. Ein Manko ist die Unterscheidung und Bewertung von Informationsangeboten nach der

1 Zum hier verwendeten Journalismus- und Medienbegriff vgl. Kohring 2000. 
Art ihrer Publikation („das“ Fernsehen vs. „die“ Zeitung) und damit an Hand eines technologischen Medienkriteriums (vgl. Robinson/Kohut 1988: 188). Hinzu kommt, dass Rezipienten unterschiedliche Konzepte für die Bewertung von Fernsehen und Zeitung heranziehen (vgl. Newhagen/Nass 1989: 284). ${ }^{2}$ Darüber hinaus entspricht die generelle Frage z. B. nach „der“ Zeitung nicht dem spezifischen Nutzungsverhalten der Rezipienten. Neben weiteren Kritikpunkten (vgl. Kohring 2001: 15 ff; 33 f) ist anzumerken, dass die Antworten nicht auf konkrete Themen bezogen werden (vgl. Meyer 1974: 49). Ein messtheoretisches Problem ergibt sich daraus, dass Glaubwürdigkeit nur an Hand eines einzigen Indikators gemessen wird, sodass nicht nur Validitäts-, sondern auch Reliabilitätseinschätzungen nicht getroffen werden können.

Im Unterschied zum Roper-Ansatz wird Glaubwürdigkeit im faktorenanalytischen Ansatz als mehrdimensionales Konstrukt aufgefasst (vgl. etwa Gaziano/McGrath 1986; Lee 1978; für einen Überblick Wirth 1999). Hierbei werden semantische Differenziale und - nur bisweilen journalismusbezogene - Items mit Hilfe explorativer Faktorenanalysen ausgewertet; die gefundenen Faktoren werden sodann als Glaubwürdigkeitsdimensionen interpretiert. So erfasste Jacobson (1969) Glaubwürdigkeit mit Hilfe eines semantischen Differenzials mit 20 Adjektivpaaren. Eine explorative Faktorenanalyse ergab neben den zwei glaubwürdigkeitsbezogenen Faktoren Authentizität und Objektivität auch zwei angeblich nicht-glaubwürdigkeitsbezogene Dimensionen, Dynamik und Ruhe.

Ganz abgesehen von dem theoretischen Einwand, dass die verwendeten Adjektivpaare zumeist nicht journalismus- und medienspezifisch sind und somit nur allgemeine Charakteristika von Kommunikation beschreiben, verdeutlicht die methodische Kritik am faktorenanalytischen Ansatz auch die generellen Probleme der explorativen Faktorenanalyse (vgl. Gorsuch 1983: 369-372). Zum Ersten ist die Annahme nicht haltbar, dass Faktoren, die in einer explorativen Studie extrahiert werden, auch die tatsächlichen Dimensionen des jeweils untersuchten Konstruktes sind. Die tatsächlichen Faktoren eines Konstruktes können nur dann als identifiziert gelten, wenn sie zum einen repliziert werden und zum anderen in einen theoretischen Rahmen eingebunden sind. Zum Zweiten verweist Gorsuch auf die Gefahr der unzureichenden Reflexion bei der Auswahl von Items. Zum Dritten berichten viele Studien nicht über die Details der Analyse, z. B. die Entscheidung über die Anzahl der Faktoren oder die Art der Rotation, was eine Replikation oder Modifikation verhindert. Auch Gorsuchs Schlussfolgerung, dass derartige Probleme auf eine mangelnde theoretische Reflexion zurückzuführen sind, gilt für die faktorenanalytische Medienglaubwürdigkeitsforschung (vgl. auch Wirth 1999: 52): In kaum einer Studie wird explizit der Versuch unternommen, das Konstrukt Glaubwürdigkeit zu definieren. ${ }^{3}$ Infolgedessen ist die Glaubwürdigkeitsdefinition für den Leser erst aus der gefundenen Faktorenlösung ersichtlich (vgl. McCroskey/Young 1979;

2 Dies führt dazu, dass Vertrauen in Journalismus auch in der Wissenschaft alltagssprachlich als Vertrauen „in Medien“ etikettiert wird. Der Einsatz technologischer Verbreitungsmedien hat zweifellos Einfluss auf die Vertrauenswürdigkeit journalistischer Informationsangebote - es ist aber theoretisch ungenau, von Vertrauen in ein technologisches Medium wie z. B. „das“ Internet oder „das“ Radio zu sprechen.

3 Vgl. etwa Al-Makaty/Boyd/Tubergen 1994; Gantz 1981; Gaziano/McGrath 1986, Jacobson 1969; Johnson/Kaye 1998; Newhagen/Nass 1989; Wanta/Hu 1994; West 1994. 
1981). ${ }^{4}$ Ein weiteres Problem ist die unzureichende Trennung von Glaubwürdigkeitsprädiktoren und der Glaubwürdigkeit selbst (vgl. Wirth 1999: 52). Schließlich wird im faktorenanalytischen Ansatz auch die Konstruktvalidität nicht thematisiert.

Der dritte, objektivitätsorientierte Ansatz verbindet das Glaubwürdigkeitskonzept mit der Objektivität journalistischer Berichterstattung. Der Ansatz geht davon aus, dass Journalisten annähernd unverzerrt über gesellschaftliche Realität berichten können und sollen. Als Beispiel für den objektivitätsorientierten Ansatz können vor allem die Arbeiten von Bentele (vgl. 1988a; 1988b; 1994a; 1994b) zur „Objektivität und Glaubwürdigkeit von Medien“ dienen (vgl. auch Nawratil 1997). Bentele zu Folge, der 1984 und 1985 in Berlin mehrere Repräsentativbefragungen durchführte, bemisst sich die journalistische Glaubwürdigkeit an der Entsprechung bzw. Diskrepanz von Medienrealität und tatsächlicher Realität (die letztlich mit der Wahrnehmung der Rezipienten gleichgesetzt wird; vgl. Bentele 1988a: 6 f).

Abgesehen von der theoretischen Diskussion, inwiefern es einen objektiven Gradmesser für die Entsprechung von journalistischer Selektivität („Medienrealität“) und Rezipientenselektivität („tatsächliche Realität“) geben kann (s. u.), ist aus methodischer Sicht die Operationalisierung des Konstrukts Glaubwürdigkeit zu kritisieren. Es wird gefragt, wie wichtig eine „wahrheitsgemäße und objektive“ (Bentele 1988a: 222; Hvhg. JM \& MK) Berichterstattung eingeschätzt wird und für „wie glaubwürdig und objektiv“ (Bentele 1988b: 415; Hvhg. JM \& MK) die einzelnen Medien gehalten werden. Bei einer solchen Fragestellung ist nicht klar, ob nur eines oder ob beide Adjektive der Antwort zu Grunde liegen. Zudem spiegelt sich Benteles (1988b: 421) Feststellung, „daß Glaubwürdigkeit nicht nur ein mehrdimensionales Konstrukt ist, sondern auch von zumindest den vier Faktorenbereichen Kommunikator/Medium, Text/Medienrealität, Rezipient und Ereignis/Realität abhängig ist“, nicht in der oben beschriebenen eindimensionalen Operationalisierung wider. Positiv hervorzuheben ist, dass die Glaubwürdigkeitseinschätzungen auf einen konkreten Themenbereich (politische Berichterstattung) und auf konkrete journalistische Medien (inkl. der von den Befragten selbst genutzten) bezogen wurden (vgl. Bentele 1988b). In späteren Arbeiten spricht Bentele (1994b, 1996, 1998) im Zusammenhang mit dem Begriff des öffentlichen Vertrauens davon, dass Glaubwürdigkeit als Teilphänomen von Vertrauen zu begreifen sei. Dieser Ansatz wird allerdings nicht weiter entwickelt.

Tabelle 1 fasst die drei Ansätze an Hand ihres Hauptcharakteristikums, des untersuchten Konstruktes, der Begriffsdefinition, des theoretischen Zugangs, der Konstruktvalidierung sowie der Methodik zusammen. Sowohl der vergleichende Ansatz als auch der faktorenanalytische Ansatz legen weder eine einheitliche Definition noch einen expliziten theoretischen Zugang vor. Neben anderen methodischen Problemen ist zu kritisieren, dass keine Konstruktvalidierung unternommen wird. Der objektivitätsorientierte Ansatz Benteles verfügt zwar über eine Definition von Glaubwürdigkeit, allerdings entspricht die gewählte eindimensionale Operationalisierung nicht der theoretischen Vorgabe. Die Darstellung verdeutlicht die beiden von Self (vgl. 1996: 434) formulierten Probleme der Glaubwürdigkeitsforschung: Weder gibt es theoretisch fundierte Skalen zur Messung des Konstruktes, noch werden mediierende Konstrukte be-

4 Dieses Vorgehen kommentieren Cronkhite und Liska (1976: 92) folgendermaßen: „It seems clear that the search for a generalized definition of credibility by these means is likely to be as costly and fruitless as the search for the Holy Grail, without any of the attendant pleasures and adventures." 
achtet. Damit wird die Konstruktvalidität von Glaubwürdigkeit resp. Vertrauen außen vor gelassen. Im nächsten Abschnitt wird ein Modell von Vertrauen in Journalismus vorgeschlagen, dass eine theoretisch fundierte Skalenkonstruktion sowie das Ableiten von Hypothesen zur Konstruktvalidierung ermöglichen soll.

\section{Tab. 1: Vergleich der Ansätze in der Medienglaubwürdigkeitsforschung}

\begin{tabular}{|c|c|c|c|}
\hline & $\begin{array}{l}\text { Vergleichender } \\
\text { Ansatz }\end{array}$ & $\begin{array}{l}\text { Faktorenanalytischer } \\
\text { Ansatz }\end{array}$ & $\begin{array}{l}\text { Objektivitäts- } \\
\text { orientierter Ansatz }\end{array}$ \\
\hline Charakteristikum & Roper-Frage & $\begin{array}{l}\text { Methode der } \\
\text { Faktorenanalyse }\end{array}$ & Objektivitätsnorm \\
\hline Konstrukt & Glaubwürdigkeit & Glaubwürdigkeit & $\begin{array}{l}\text { Glaubwürdigkeit } \\
\text { (später auch Vertrauen) }\end{array}$ \\
\hline Begriffsdefinition & $\begin{array}{l}\text { keine einheitliche } \\
\text { Definition vorhanden }\end{array}$ & $\begin{array}{l}\text { keine einheitliche } \\
\text { Definition vorhanden }\end{array}$ & $\begin{array}{l}\text { Definition für Glaub- } \\
\text { würdigkeit vorhanden }\end{array}$ \\
\hline $\begin{array}{l}\text { Theoretischer } \\
\text { Zugang }\end{array}$ & nicht expliziert & nicht expliziert & $\begin{array}{l}\text { normative } \\
\text { Journalismustheorie }\end{array}$ \\
\hline Konstruktvalidität & $\begin{array}{l}\text { keine Hypothesen } \\
\text { formuliert }\end{array}$ & $\begin{array}{l}\text { keine Hypothesen } \\
\text { formuliert }\end{array}$ & $\begin{array}{l}\text { Hypothesen ableitbar, } \\
\text { aber nicht überprüft }\end{array}$ \\
\hline Methodik & $\begin{array}{l}\text { methodische Mängel } \\
\text { bei der } \\
\text { Operationalisierung } \\
\text { der Roper-Frage }\end{array}$ & $\begin{array}{l}\text { methodische Mängel } \\
\text { beim Einsatz der } \\
\text { Faktorenanalyse }\end{array}$ & $\begin{array}{l}\text { Operationalisierung } \\
\text { entspricht nicht } \\
\text { der theoretischen } \\
\text { Vorgabe }\end{array}$ \\
\hline
\end{tabular}

\section{Ein Faktorenmodell von Vertrauen in Journalismus}

Die methodischen Defizite des vergleichenden und des faktorenanalytischen Ansatzes gehen einher mit journalismus- und vertrauenstheoretischen Auslassungen: „Das zentrale Problem ist zweifellos die Theorielosigkeit des Vorgehens." (Wirth 1999: 52) Der objektivitätsorientierte Ansatz verfügt als einziger zwar über eine ausgearbeitete Journalismustheorie, hat aber bislang noch keinen Operationalisierungsvorschlag für die Frage, woran sich Objektivität denn bemessen soll, vorlegen können. Bentele versucht, dieses Problem normativ mit Hilfe eines Objektivitätspostulats zu lösen (vgl. Bentele 1994: 305), das sich auch auf die journalistische Selektivität erstreckt und im Übrigen nur für den informierenden Journalismus gelten soll. Diese Norm einer kritischen Objektivität setzt allerdings die Vorstellung einer außermedialen Realität voraus, mit der die Berichterstattung verglichen werden kann. Sofern man nicht behaupten will, dass diese außermediale Realität an sich vorhanden sei, kann man die journalistische Sicht der Dinge nur mit der anderer gesellschaftlicher Akteure vergleichen. Solche außermedialen Realitätssichten sind aber selbst hochselektiv und können nicht zum primären Maßstab journalistischen Handelns gemacht werden, sofern man auf dessen Autonomie Wert legt. Ohne das Problem der normativen Bewertung journalistischer Informationsleistungen damit ignorieren zu wollen, wird an dieser Stelle der theoretischen Vorstellung der Vorzug gegeben, dass es erstens nicht möglich ist, journalistische Informationsan- 
gebote mit einer beobachterunabhängigen nicht-journalistischen Realität zu vergleichen, und dass zweitens gerade aus diesem Grunde die Autonomie journalistischer Selektivität die Basis für dessen gesellschaftliche Funktionserfüllung darstellt. Beide Gründe zusammen verdeutlichen, warum es riskant ist, sich auf journalistische Informationen zu verlassen. Nur deshalb ist es aber überhaupt sinnvoll und notwendig, über Vertrauen in Journalismus zu reden.

Eine zweite theoretische Vorbemerkung gilt dem Vertrauens- resp. Glaubwürdigkeitsbegriff. In der Forschungsliteratur wird Glaubwürdigkeit zum einen als Glauboder Wahrhaftigkeit (believability) verstanden, was wieder auf den erkenntnistheoretisch als unzulänglich erkannten Vergleich von journalistischer mit außermedialer Realität hinausläuft. Zum anderen wird auf die Formel von Hovland, Janis und Kelley (vgl. ${ }^{3} 1959$ ) zurückgegriffen, die die Glaubwürdigkeit eines Kommunikators als Produkt von Vertrauenswürdigkeit (verstanden als Aufrichtigkeit) und Kompetenz behaupteten, ohne diese Entscheidung weiter zu begründen. Insgesamt ist zu konstatieren, dass die Forschung nicht über einen theoretisch ausgearbeiteten Glaubwürdigkeits- oder Vertrauensbegriff verfügt. Es ist daher notwendig, einen Vertrauensbegriff einzuführen, der hinreichend abstrakt ist, um auf die oben beschriebene Problematik riskanten, da unweigerlich selektiven journalistischen Handelns spezifisch zugeschnitten werden zu können. Im Folgenden wird daher der teilweise noch weiter explizierte Vertrauensbegriff von Niklas Luhmann zu Grunde gelegt (vgl. Luhmann 1989; Kohring 2001: 65 ff), der hierfür als am leistungsfähigsten erachtet wird.

Der theoretische Ausgangspunkt für die Analyse von Vertrauen in (medial vermittelten) Journalismus ist nicht Objektivität oder Wahrheit, sondern Selektivität, anders ausgedrückt: Vertrauen in Journalismus ist Vertrauen in dessen spezifische Selektivität. Dass diese kontingent ist, macht es prinzipiell riskant, dem eigenen Handeln journalistische Informationsangebote zu Grunde zu legen (so wie es prinzipiell immer riskant ist, sich in die Hände Anderer zu begeben). Diese als Risiko wahrgenommene Ungewissheit einer komplexen Zukunft wird durch Vertrauen kompensiert. Vertrauen „überzieht die Informationen, die es aus der Vergangenheit besitzt, und riskiert eine Bestimmung der Zukunft. Im Akt des Vertrauens wird die Komplexität der zukünftigen Welt reduziert. Der vertrauensvoll Handelnde engagiert sich so, als ob es in der Zukunft nur bestimmte Möglichkeiten gäbe.“ (Luhmann 1989: 20) Vertrauen fungiert dabei als Ersatz für Wissen: Im Moment der Vertrauenshandlung - ,als Hypothese ein mittlerer Zustand zwischen Wissen und Nichtwissen“ (Simmel¹999: 393) - weiß der Vertrauende nicht, ob sein Vertrauen berechtigt ist. Vertrauen wird daher definiert als „selektive Verknüpfung von Fremdhandlungen mit Eigenhandlungen unter der Bedingung einer rational nicht legitimierbaren Tolerierung von Unsicherbeit." (Kohring 2001: 67)

Das hier zu Grunde gelegte Modell von Vertrauen in Journalismus (vgl. Kohring 2002a; 2002b) geht gesellschafts- und journalismustheoretisch davon aus, dass Journalismus die in viele spezialisierte Teilbereiche ausdifferenzierte Gesellschaft stets daraufhin beobachtet, ob Ereignisse in einem Bereich Resonanz in anderen Bereichen der Gesellschaft auszulösen vermögen. Journalismus beobachtet die Gesellschaft im Hinblick auf die Mehrsystemzugehörigkeit von Ereignissen: Er kommuniziert ausschließlich über solche Ereignisse, die über den gesellschaftlichen Bereich hinaus, in dem sie passiert sind, Bedeutung erlangen könnten (vgl. Kohring 1997; 2000: 163 ff). Diese Beobachtungsleistungen des Journalismus entlasten die anderen gesellschaftlichen Systeme von der Notwendigkeit, ihre Umwelt-Erwartungen ständig selbst zu überprüfen, um sie gegebenenfalls zu modifizieren. Das spezifische Publikumsvertrauen in Journalismus richtet sich also darauf, dass seine Informationsangebote tatsächlich eine solche Orien- 
tierung ermöglichen. Orientierung bedeutet, dass journalistische Selektionen in die Abfolge eigener Selektionen quasi eingebaut werden und sie sowohl begründen als auch überhaupt erst ermöglichen. ${ }^{5}$ Auf der Grundlage dieser Überlegungen wurden vier Dimensionen journalistischer Selektivität abgeleitet, deren Zusammenspiel, so die Hypothese, das Konstrukt Vertrauen in Journalismus ausmacht. Alle vier Faktoren leiten sich also aus der oben skizzierten Funktion von Journalismus ab und beziehen sich in unterschiedlicher Weise auf die damit verbundene spezifische journalistische Selektivität. Wenn Rezipienten Vertrauen in Journalismus fassen, so die Annahme, treffen sie ihre Vertrauensbewertungen auf den folgenden vier Dimensionen (vgl. ausführlicher Kohring 2001: $85 \mathrm{ff}$ ):

\section{1) Vertrauen in Themenselektivität}

Die Auswahl von Themen der Berichterstattung markiert die erste Vertrauensdimension. Die Rezipienten vertrauen darauf, dass sie vom Journalismus auf diejenigen Themen und Ereignisse aufmerksam gemacht werden, die für sie relevant sind.

2) Vertrauen in Faktenselektivität

Hier geht es um die Auswahl von Fakten oder Hintergrundinformationen zu einem bereits selektierten Thema, also um die Art und Weise, wie ein Ereignis kontextualisiert wird.

3) Vertrauen in die Richtigkeit von Beschreibungen (Glaubwürdigkeit)

Hierunter fällt das Vertrauen in die nachprüfbare und somit konsentierbare Richtigkeit der dargestellten Fakten. Zwar sind Beobachtungen hochselektiv und ihre Unterteilung in „richtige“ oder "falsche“ nicht objektiv bestimmbar. Allerdings gibt es auch eine Reihe von Beobachtungen, bei denen die Unterscheidung zwischen „richtig“ und „falsch“ normiert und daher nachprüfbar ist. Glaubwürdigkeit wird damit als ein Teilkonzept von Vertrauen in Journalismus verstanden.

4) Vertrauen in journalistische Bewertungen

Bereits die Selektion eines Ereignisses oder von Informationen stellt eine Bewertung dar. Hinzu kommen explizit hervorgehobene Bewertungen, vor allem als Kommentare. Sie geben Hinweise, Handlungsbewertungen und Handlungsaufforderungen.

Für die Operationalisierung des hier vorgeschlagenen Faktorenmodells erschien es sinnvoll, ein qualitatives Vorgehen mit einem quantitativen zu verbinden (vgl. Kelle/Erzberger 1999; Tashakkori/Teddlie 1998). Auf diesem Wege können nicht nur die Itemformulierung optimiert und die Hypothesenentwicklung stimuliert, sondern auch unterschiedliche Aspekte von Vertrauen beleuchtet werden. Folglich wurden in einem ersten Schritt im Januar/Februar 2001 mit 27 Personen qualitative Leitfadengespräche durchgeführt, die gezielt das oben skizzierte theoretische Konzept umsetzten (vgl. Matthes 2001: 64-83). Aus den Interviews wurde ein Itempool mit 50 Statements generiert. Mittels offener Fragen wurden die Erwartungen der Befragten an die Medienberichterstattung sowie die Relevanz und die Einschätzung der vier Vertrauensfaktoren exploriert. Die inhaltsanalytische Auswertung der Interviews ergab, dass die Befragten die vier Dimensionen von Vertrauen in Journalismus tatsächlich erwarten, wahrnehmen und differenzieren. Die Interviews lieferten zudem Hinweise für eine neue potenzielle Subdimension, die man als Vollständigkeit von Informationen bezeichnen könnte. Vollständigkeit meint, dass alle wichtigen Informationen zu einem Thema bereitgestellt werden und die Befragten sich ausreichend informiert fühlen. Die Vollständigkeit von In-

5 So z. B., wenn man die Börsenberichterstattung der $F A Z$ zur Grundlage des eigenen Aktiengeschäfts nimmt. 
formationen ist einerseits als Subdimension von Faktenselektivität vorstellbar. Da für manche Befragte schon das Weglassen von wichtigen Informationen eine Verfälschung oder Lüge darstellt, könnte die Vollständigkeit von Informationen andererseits aber auch eine Subdimension von Glaubwürdigkeit sein. ${ }^{6}$ Eine Antwort auf diese Frage sollte die quantitative Befragung liefern.

\section{Hypothesen und methodisches Vorgehen}

Auf der Basis des in Abschnitt 3 skizzierten theoretischen Modells von Vertrauen in Journalismus sowie auf Grund der qualitativen Vorstudie werden im Folgenden Hypothesen für eine standardisierte Befragung abgeleitet. Zunächst gilt es zu überprüfen, ob die befragten Individuen ihr Vertrauen in die Medien an den vier postulierten Vertrauensfaktoren ausrichten:

\section{Hypothese 1 \\ Vertrauen in Journalismus ist ein vierdimensionales Konstrukt, das sich aus den in- terdependenten Faktoren „Vertrauen in Themenselektivität“, „Vertranen in Fakten- selektivität“, „Vertranen in die Richtigkeit von Beschreibungen“"(Glaubwürdigkeit) und „Vertrauen in Bewertungen“ zusammensetzt.}

Die sekundäre Zielstellung besteht in einer ersten Konstruktvalidierung der Vertrauensskala. Der Prozess der Validierung - als die wichtigste Herausforderung an die sozialwissenschaftliche Skalenentwicklung - kann allgemein als kumulative Beweisführung zur Etablierung eines Messinstrumentes verstanden werden. Während die Faktorenanalyse auf eine strukturelle Validierung abzielt, erfolgt bei der Konstruktvalidierung ein sukzessives Überprüfen von aus einem theoretischen Netzwerk abgeleiteten Hypothesen (John/Benet-Martínez 2000: 351; Bryant 2000: 112). Ohne die Konstruktvalidierung einer Skala ist nicht sichergestellt, was die Skala wirklich erfasst. Für dieses Vorhaben wurde zunächst in einem ersten Schritt die Persönlichkeitseigenschaft Vertrauensfähigkeit extrahiert. Nach Luhmann (vgl. 1989: 85-94) wird der Vertrauenserweis durch strukturell nicht gebundene interne Ressourcen wie die generelle Vertrauensfähigkeit ermöglicht und erleichtert (vgl. Kohring 2001: 63-64). Diese Fähigkeit liegt in einer „Steigerung tragbarer Unsicherheit auf Kosten von Sicherheit“ (Luhmann 1989: 88). Vertrauensfähigkeit ist eine Persönlichkeitseigenschaft, der gemäß dem handlungstheoretischen Partialmodell der Persönlichkeit von Krampen (1997: 40-41) vor allem in „subjektiv mehrdeutigen, neuartigen und kognitiv schlecht strukturierbaren Handlungs- und Lebenssituationen eine hohe Bedeutung für Verhalten und Erleben zukommt". Vertrauensfähigkeit wird also in als kontingent wahrgenommenen Situationen relevant. „Mit zunehmender Komplexität [steigt] auch der Bedarf für Vergewisserungen der Gegenwart, zum Beispiel für Vertrauen“ (Luhmann 1989: 13). Für eine erste Konstruktvalidierung von Vertrauen in Journalismus können daher die folgenden Annahmen abgeleitet werden:

6 Für Bentele (vgl. 1994a: 309) stellen Vollständigkeit und Richtigkeit die grundlegenden Merkmale journalistischer Objektivität dar. Objektivität im Sinne Benteles wäre hier einer von vier Faktoren journalistischer Vertrauenswürdigkeit. 
Hypothesen 2a, 2b, 2c, $2 d$

Je höher die Vertrauensfähigkeit einer Person ist, desto größer ist ibr Vertrauen in die:

2a) journalistische Themenselektivität,

2b) journalistische Faktenselektivität,

2c) Richtigkeit journalistischer Beschreibungen (Glaubwürdigkeit),

2d) journalistischen Bewertungen.

Die qualitative Vorstudie lieferte Hinweise auf eine zusätzliche Subdimension, die als Vollständigkeit von Informationen bezeichnet wurde. Offen blieb die Frage, ob diese Subdimension dem Faktor Faktenselektivität oder dem Faktor Richtigkeit von Beschreibungen zuzuordnen ist. Daraus ergibt sich die folgende Fragestellung:

\section{Fragestellung 1 \\ Handelt es sich bei „Vollständigkeit"von Informationen um eine Subdimension von „Faktenselektivität" oder um eine Subdimension von „Richtigkeit von Beschrei- bungen"(Glaubwürdigkeit)?}

Da vor allem Hypothese 1 eng mit dem statistischen Verfahren der Faktorenanalyse verknüpft ist, und da die Handhabung dieses Verfahrens eine entscheidende Rolle im Prozess der Skalenentwicklung spielt, soll an dieser Stelle kurz die faktorenanalytische Auswertungsstrategie vorgestellt werden. Für die Überprüfung von Hypothese 1 ist das bislang in der Forschung fast ausschließlich verwendete Verfahren der explorativen Faktorenanalyse (EFA) nur bedingt geeignet, da es keine Aussage über die Gültigkeit der gefundenen Struktur erlaubt. Die Struktur des Vertrauens sollte daher mit einer konfirmatorischen Faktorenanalyse (KFA) empirisch überprüft und gegebenenfalls modifiziert werden. Sowohl die EFA als auch die KFA können als Datenreduktionstechniken verstanden werden, die Kovarianzen zwischen mehreren beobachteten Variablen (Indikatoren) und wenigen zugrundeliegenden latenten Variablen (Faktoren oder Dimensionen) erklären (vgl. Hoyle 2000). In der Aufdeckung noch nicht bekannter Faktorstrukturen liegt die Stärke der EFA. Bei der KFA hingegen (als einer Anwendungsmöglichkeit so genannter Strukturgleichungsmodelle) werden - vereinfacht ausgedrückt - „kausale“ Beziehungen zwischen latenten und manifesten Variablen überprüft. Im Gegensatz zur EFA, bei der jedes Item auf jedem Faktor frei laden kann, wird bei der KFA ein Messmodell spezifiziert, das die jeweiligen Indikatoren einzelnen Faktoren zuordnet. Weiterhin ermöglicht die KFA die Berücksichtigung von Messfehlern und die Schätzung von Anpassungsmaßen für verschiedene Teile des Faktorenmodells. Der grundlegende Vorteil der KFA besteht demnach darin, dass a priori generierte Hypothesen über die genaue Zuordnung der Indikatoren zu den Faktoren überprüft und falsifiziert werden können (vgl. Schumaker/Lomax 1996: 45-47).

Die hier vorgestellte theoretische Unterscheidung von vier Vertrauensfaktoren legt den Einsatz einer KFA nahe. Zusätzlich wird aus folgenden Gründen eine EFA vorgeschaltet: Zunächst erlaubt die EFA, die am besten geeigneten Items für die postulierten Dimensionen zu bestimmen. Die Verwendung einer EFA ermöglicht darüber hinaus das Erkennen von neuen Gesichtspunkten oder Besonderheiten einer Skala. Gerbing und Hamilton (1996) konnten in einer Simulationsstudie zeigen, dass sich die EFA als Vorstufe zur KFA eignet und schlagen deshalb vor, die Stärken beider Verfahren im Prozess der Skalenkonstruktion sinnvoll zu verbinden (vgl. ebd.: 63; Hoyle 2000: 469). Ausgehend von diesen Überlegungen kann das faktorenanalytische Vorgehen folgen- 
dermaßen beschrieben werden: Zunächst wird eine EFA durchgeführt, die vor allem der Auswahl der besten Items dient. Im Anschluss wird eine KFA nach den vier postulierten Faktoren spezifiziert. Auf Grund des somit teilweise datengeleiteten Vorgehens besteht die Notwendigkeit, das Modell in einer zukünftigen Studie zu prüfen und zu validieren.

\section{Ergebnisse}

Die durch die qualitative Vorstudie gewonnenen Items flossen in eine standardisierte schriftliche Befragung ${ }^{7}$ mit einer anfallenden Stichprobe von 303 (48\% weiblich / 52\% männlich) erwachsenen Personen ein (vgl. Matthes 2001). Die Teilnehmer beantworteten Fragen zu der Berichterstattung über die neue Währung „Euro“. Das Alter der Befragten variierte von 15 bis 76 Jahren (Durchschnitt: 30,8). Verzerrungen können bei einer solchen Stichprobenziehung nicht ausgeschlossen werden. Allerdings sind in dieser Studie verschiedene soziodemographische Segmente vertreten (43\% Berufstätige, 37\% Studierende, 10\% Arbeitslose, 6\% Auszubildende, 4\% Rentner). Zumindest hinsichtlich Alter und Bildung ist damit eine angemessene Streuung gewährleistet.

\subsection{Konstruktion der Vertranensskala}

Die Items wurden zunächst mit einer EFA ausgewertet. Das Ergebnis entspricht den postulierten vier Faktoren von Vertrauen in Journalismus. ${ }^{8}$ Überdies zeigt sich, dass anscheinend die Vollständigkeit von Informationen unter den Faktor Richtigkeit von Beschreibungen resp. Glaubwürdigkeit subsumiert werden kann: Sowohl die Items, die sich theoretisch dem Faktor Vollständigkeit von Informationen zuordnen lassen, als auch die abgeleiteten Items zur Richtigkeit von Beschreibungen bilden einen gemeinsamen, sehr robusten Faktor, der sich in verschiedenen Analysen immer wieder zeigte. In der KFA wird auf Grund dieses Ergebnisses ein hierarchischer Faktor Richtigkeit von Beschreibungen resp. Glaubwürdigkeit spezifiziert, der die zwei Subdimensionen Korrektheit von Informationen und Vollständigkeit von Informationen aufweist. Hierfür ließen sich ja bereits in der qualitativen Vorstudie Hinweise finden. ${ }^{9}$ Ziel einer KFA ist es nun zu überprüfen, wie gut die gesamte angenommene Struktur den empirischen Daten entspricht.

In einer hierarchischen KFA mit Maximum-Likelihood-Schätzung (vgl. Rindskopf/Rose 1988; Bollen 1989: 313-315) wurde ein Modell mit den vier Faktoren spezifiziert, wobei einer dieser Faktoren, Richtigkeit von Beschreibungen (Glaubwürdig-

7 Die Befragung wurde im August 2001 in der Innenstadt von Jena durchgeführt.

8 Auf Grund der postulierten Abhängigkeit der Faktoren untereinander wurde schiefwinklig rotiert (Oblimin). Die Extraktion der Faktoren erfolgte über das Kaiserkriterium. Ausschlusskriterium für Items war eine Ladung < .3, Doppelladung oder das Aufspannen eines nicht interpretierbaren Faktors. Die gewonnene Faktorlösung erklärt 57\% der Gesamtvarianz. Die Ergebnisse können aus Platzgründen nicht gesondert berichtet werden. Die EFA führte u. a. zum Ausschluss von mehreren negativ formulierten Items, die einen gemeinsamen Faktor bildeten.

9 Es ist ausdrücklich zu betonen, dass die Subdimension „Vollständigkeit von Informationen“ von der EFA nicht erst neu entdeckt wurde, da sie gezielt in die Fragebogenkonstruktion eingeflossen ist. Jedes Item war schon vor der EFA eindeutig einem Faktor zugeordnet. Durch die EFA wurden lediglich die besten Items für jeden Faktor ausgewählt. 


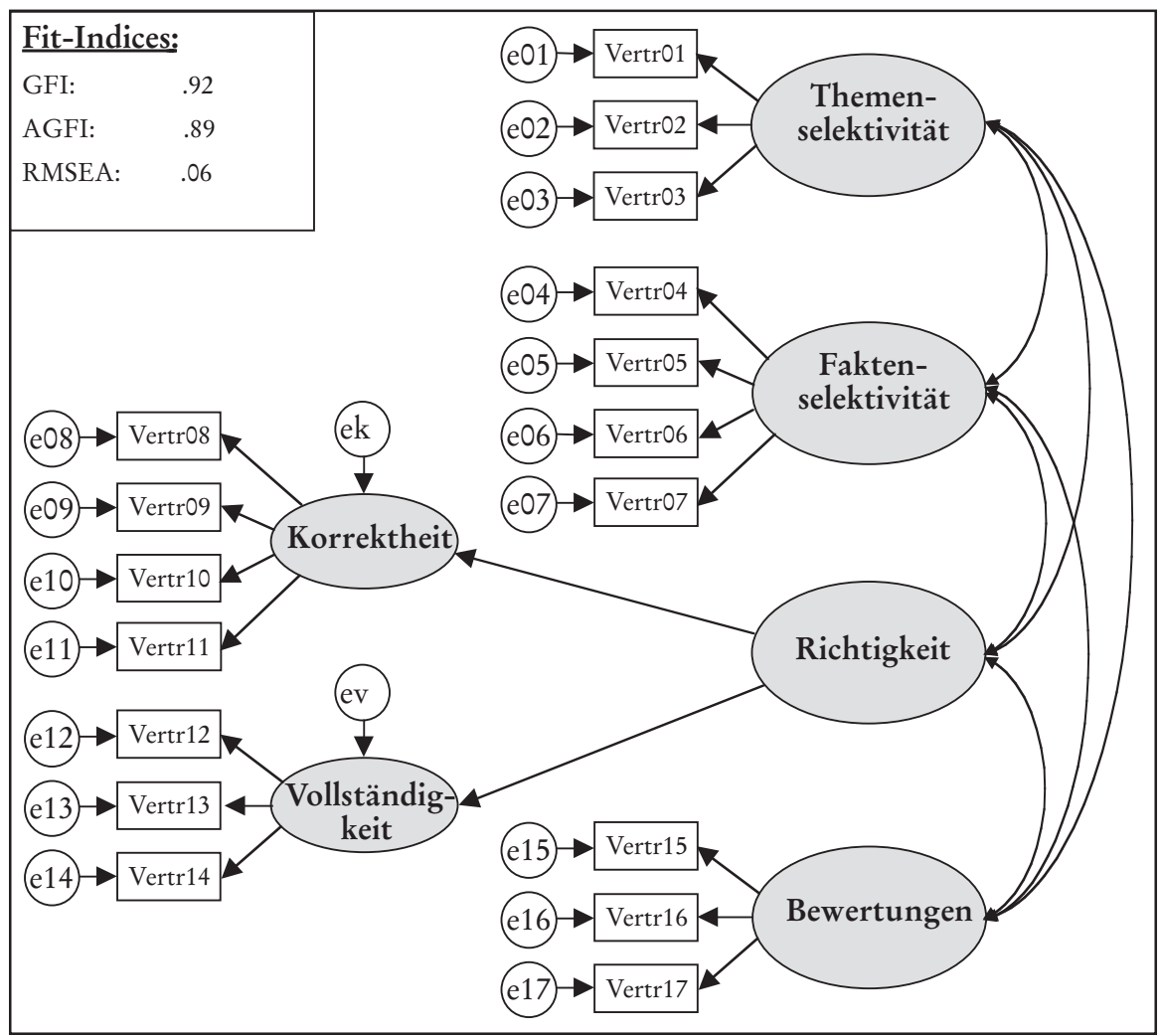

keit), zwei Subdimensionen bzw. Faktoren erster Ordnung umfasst: Korrektheit von Informationen und Vollständigkeit von Informationen. ${ }^{10}$ Im Rahmen der Modellmodifizierung kam es zu einem weiteren Ausschluss von sechs Items. Als Ausschlusskriterium dienten hierbei korrelierte Messfehler oder ungewollte Doppelladungen eines Items auf einem fremden Faktor (vgl. zu diesem Vorgehen Kline 1998: 216-219). Dies führte schließlich zu dem in Abbildung 1 gezeigten Faktorenmodell. Die großen Kreise stehen für den jeweiligen latenten Faktor, die Kästchen für das entsprechende Item und die klei-

10 Um die latenten Konstrukte zu skalieren, wurden die Varianzen der vier Faktoren gemäß dem gängigen Vorgehen auf 1 fixiert, für die beiden Subdimensionen wurde jeweils eine Indikatorladung auf 1 gesetzt (vgl. Kline 1998: 203-207). Ebenfalls wurde die für Strukturgleichungsmodelle notwendige Annahme auf Normalverteilung überprüft (vgl. Hoyle/Panter 1995: 162). Die Stichprobengröße von $\mathrm{n}=303$ entspricht den Mindestanforderungen für eine KFA (vgl. Hoyle 2000: 472). Fehlende Werte wurden standardmäßig durch den Mittelwert aller Befragten ersetzt, wobei der maximale Anteil fehlender Werte zu einem Item bei 3\% lag. 
nen Kreise für den dazugehörigen Messfehler. Die doppelseitigen Pfeile zwischen den Faktoren bezeichnen die Interfaktorkorrelationen und einseitige Pfeile die Regressionsgewichte bzw. die Faktorladungen. Die Fit-Indices GFI (Goodness of Fit) und AGFI (Adjusted Goodness of Fit) genügen dem Anspruchsniveau von $\geq .9$ (vgl. Homburg/Pflesser 2000: 430; für einen Überblick siehe Hu/Bentler 1999). Der RMSEA-Wert (root mean squared error of approximation) genügt ebenfalls dem Anspruchsniveau von $<.08$ (vgl. Homburg/Pflesser 2000: 430). Die postulierte Faktorstruktur (Hypothese 1) kann somit als vorläufig bestätigt gelten: Die Befragten unterscheiden die vier dargestellten Faktoren, wenn sie ihr Vertrauen in Journalismus äußern.

Die verhältnismäßig hohen Korrelationen $(\phi)$ zeigen, dass die Faktoren gemäß der Hypothese nicht unabhängig voneinander $\operatorname{sind}^{11}$ :

\section{Themenselektivität \\ Themenselektivität \\ Themenselektivität \\ Richtigkeit \\ Richtigkeit \\ Faktenselektivität}

$\begin{array}{ll}\leftrightarrow & \text { Faktenselektivität: } \\ \leftrightarrow & \text { Bewertung: } \\ \leftrightarrow & \text { Richtigkeit: } \\ \leftrightarrow & \text { Bewertung: } \\ \leftrightarrow & \text { Faktenselektivität: } \\ \leftrightarrow & \text { Bewertung: }\end{array}$$$
\begin{aligned}
& \phi=.71 \\
& \phi=.65 \\
& \phi=.78 \\
& \phi=.75 \\
& \phi=.82 \\
& \phi=.82
\end{aligned}
$$

Auf Grund der hohen Korrelationen zwischen den Faktoren wurden zusätzlich drei alternative Modellstrukturen an den Daten überprüft. Zum Ersten wurden die beiden Subdimensionen zu einem nicht-hierarchischen Faktor (Richtigkeit von Beschreibungen mit sieben Items) zusammengefasst, d. h. es wurde nicht mehr zwischen Vollständigkeit und Korrektheit unterschieden. In einer weiteren hierarchischen Analyse wurden die Vollständigkeit von Informationen und der zuvor allein stehende Faktor Faktenselektivität als zwei Subdimensionen einer neuen latenten Variable betrachtet, die vorherige Subdimension Korrektheit von Informationen wurde hierbei dann als eigenständiger Faktor spezifiziert. Schließlich wurde ein hierarchisches Modell spezifiziert, in dem ein einziger latenter hierarchischer Faktor (Vertrauen) alle vier Faktoren erster Ordnung erklärt. Alle drei alternativen Modelle wurden auf der Basis der oben beschriebenen Anspruchsniveaus den Fit-Indices nicht gerecht.

Im Folgenden werden die Faktoren mit den dazugehörigen Items und den Faktorladungen $(\lambda)$ vorgestellt. In Klammern ist jeweils die Reliabilität (R) angegeben. Auf Grund der eingeschränkten Aussagekraft des gängigen Koeffizienten Cronbach's Alpha (vgl. Homburg/Giering 1998: 120) wurde die Faktorreliabilität als durchschnittlich erfasste Varianz eines Faktors berechnet (ebd.: 124). Das Anspruchsniveau beträgt R > .60 (vgl. Homburg/Pflesser 2000: 430).

11 Die Höhe der Korrelationen macht allerdings eine statistische Überprüfung der Diskriminanzvalidität notwendig. Homburg/Giering (vgl. 1998: 126) schlagen hierfür den $\chi^{2}$-Differenztest vor. Hierbei wird der Wert der $\chi^{2}$-Differenz zwischen dem ursprünglichen, tatsächlich berechneten Modell und einem Modell, bei dem die Korrelation zwischen zwei Faktoren auf 1 fixiert wird, berechnet. Bei einer signifikanten Verschlechterung des $\chi^{2}$-Wertes ist von Diskriminanzvalidität auszugehen. Dies ist bei allen hier untersuchten Faktoren der Fall. 
Faktor 1: Vertrauen in die Themenselektivität $(R=.55)$

Vertr01 „Dem Thema wird die Aufmerksamkeit geschenkt, die angebracht ist.“ $(\lambda=.78)$

Vertr02 „Die Häufigkeit, mit der über dieses Thema berichtet wird, ist angemessen.“ $(\lambda=.63)$

Vertr03 „Es wurde zu spät auf dieses Thema aufmerksam gemacht.“ $(\lambda=-.38)$

Dieser Faktor beschreibt das Vertrauen der Befragten in die Thematisierungsfunktion der Medien. Hierunter fallen drei Aspekte: Zum Ersten wird gemessen, wie ausführlich ein Thema (hier: der Euro) in der Berichterstattung berücksichtigt wird. Ein damit eng verknüpfter zweiter Aspekt, der in der Itemformulierung berücksichtigt wurde, ist die Häufigkeit der Berichterstattung. Der Zeitpunkt der Berichterstattung ist ein dritter Aspekt der hier vorgenommenen Operationalisierung. ${ }^{12}$

\section{Faktor 2: Vertrauen in die Faktenselektivität $(R=.69)$}

Vertr04 „Es werden verschiedene Aspekte dieses Themas angesprochen.“ $(\lambda=.72)$

Vertr05 „Das Thema wird von mehreren Seiten beleuchtet.“ $(\lambda=.72)$

Vertr06 „Die Berichterstattung erfolgt nicht nur aus einem Blickwinkel, sondern es wird immer auch die Gegenseite gezeigt." $(\lambda=.64)$

Vertr07 „Es stehen unwichtige Dinge im Vordergrund.“ $(\lambda=-.44)$

Dieser Faktor bezeichnet das Vertrauen in die Art und Weise, wie ein Thema dargestellt wird. Beim Thema Euro bedeutet das, ob verschiedene Seiten des Themas - beispielsweise wirtschaftliche, gesellschaftliche oder soziale Aspekte - vom Journalismus angesprochen werden. Hierunter fällt auch die Ausgewogenheit der Berichterstattung, also ob ein Thema nur einseitig oder aus mehreren Perspektiven dargestellt wird.

\section{Faktor 3: Vertrauen in Richtigkeit von Beschreibungen (Glaubwürdigkeit)}

Der Faktor Richtigkeit von Beschreibungen resp. Glaubwürdigkeit (Faktor zweiter Ordnung) wird hier als ein Konstrukt verstanden, das durch die zwei Subdimensionen (Faktoren erster Ordnung) Korrektheit von Informationen und Vollständigkeit von Informationen repräsentiert wird. Dies ist bei Strukturgleichungsmodellen dann üblich, wenn die entsprechenden Faktoren erster Ordnung eine hohe bis sehr hohe Korrelation aufweisen (vgl. Byrne 2001: 120 ff; Kline 1998: 233 ff). Auch in diesem Modell wird die sehr hohe Korrelation der beiden Faktoren erster Ordnung durch eine gemeinsame Ursache erklärt, den Faktor Richtigkeit von Beschreibungen. Die direkten Effekte $(\gamma)$ dieses Faktors auf die beiden Subdimensionen Korrektheit von Informationen $(\gamma=.98)$

12 Auffallend ist bei diesem Faktor zunächst die geringe Faktorladung des dritten Items und die dadurch verursachte geringe Faktorreliabilität. Die schwache Faktorladung kommt möglicherweise durch die negative Itemformulierung zustande. Würde man dieses Item entfernen, stiege nicht nur die Faktorreliabilität von Themenselektivität, sondern auch die gesamte Anpassungsgüte des Modells. Es wurde aber gegen einen Ausschluss des Items entschieden, da es inhaltlich sehr gut zu dem Faktor passt. Mit anderen Worten, die Validität bzw. die inhaltliche Komplexität des Faktors soll an dieser Stelle nicht der Reliabilität zum Opfer fallen. 
und Vollständigkeit von Informationen $(\gamma=.95)$ sind ebenfalls sehr hoch, sodass nur ein sehr geringer Anteil der Varianz der beiden Subdimensionen nicht erklärt ist. Im Folgenden werden die Items der beiden Subdimensionen mit den Faktorladungen $(\lambda)$ aufgeführt.

\section{Subdimension 1: Vertrauen in Korrektheit von Informationen $(R=.76)$}

Vertr08 „Die Berichte geben die Dinge so wieder, wie sie sind.“ $(\lambda=.77)$

Vertr09 "Ich kann mich auf die Informationen verlassen.“ $(\lambda=.73)$

Vertr10 „Ich erhalte korrekte Informationen, die ich an andere weitergeben kann.“ $(\lambda=.70)$

Vertr11 „Es wird die ganze Wahrheit berichtet.“ $(\lambda=.63)$

\section{Subdimension 2: Vertrauen in Vollständigkeit von Informationen $(R=.81)$}

Vertr12 „Es werden mir genau die Informationen geboten, die ich brauche. “ $(\lambda=.85)$

Vertr13 „Ich erhalte alle Hintergrundinformationen, die ich benötige. “ $(\lambda=.79)$

Vertr14 „Durch die Berichterstattung kann ich mir ein vollständiges Bild über das Thema machen." $(\lambda=.77)$

Der Faktor Korrektheit von Informationen spiegelt den Eindruck der Befragten wider, inwieweit die berichteten Fakten der subjektiv empfundenen Wahrheit entsprechen. Vollständigkeit von Informationen bezieht sich hingegen darauf, ob zu einem Thema alle wichtigen Fakten genannt werden. Offenbar ist das Weglassen wichtiger Informationen schon mit einer (Ver-)Fälschung gleichzusetzen. Möglicherweise unterstellen die Rezipienten hierbei ein bewusstes Unterschlagen von Informationen.

\section{Faktor 4: Vertrauen in journalistische Bewertungen $(R=.66)$}

Vertr15 „Die Kommentare der Journalisten sind hilfreich.“ $(\lambda=.81)$

Vertr16 „Wenn die Journalisten Kritik äußern, tun sie das in angemessener Weise.“ $(\lambda=.59)$

Vertr17 „Es ist sehr interessant, wenn die Journalisten ihre Meinung zu diesem Thema äußern.“ $(\lambda=.45)$

Der Faktor Vertrauen in journalistische Bewertungen bezieht sich in der hier gewählten Operationalisierung auf explizite Bewertungen der Journalisten. Hierunter fallen nicht nur die Kritikfunktion der Medien, sondern auch Handlungsanweisungen, die dem Befragten helfen, sich zu orientieren oder Entscheidungen zu treffen. Die Faktorreliabilitäten entsprechen - mit Abstrichen beim Faktor Themenselektivität - dem gängigen Anspruchsniveau. Auch die Reliabilität von Vertrauensfähigkeit (Cronbach's Alpha = $.80 ; 3$ Items) ist zufrieden stellend. Dies ermöglicht in einem nächsten Schritt die Überprüfung der Hypothesen $2 \mathrm{a}-2 \mathrm{~d}$.

\subsection{Konstruktvalidierung}

Einem Vorschlag von Krampen (vgl. 1997: 39) folgend wurden für die Erfassung von Vertrauensfähigkeit Items aus der standardisierten H-RA-Skala zur Messung von Hoffnungslosigkeit (bzw. Zukunftsvertrauen) verwendet (vgl. Krampen 1994). Für jeden der 
Abb. 2: Strukturgleichungsmodell zur Erklärung von Vertrauen in Themenselektivität mit Fit-Indices

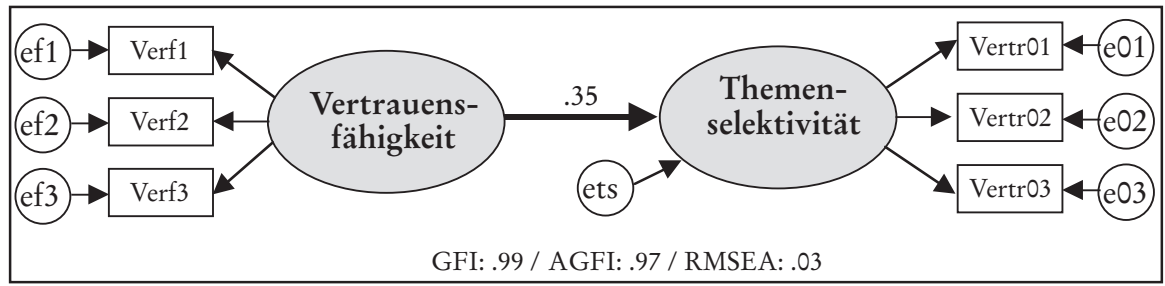

vier Vertrauensfaktoren wurde ein Strukturgleichungsmodell spezifiziert, in dem ausschließlich die Hypothesen zum Zusammenhang von Vertrauensfähigkeit und dem jeweiligen Vertrauensfaktor getestet wurden. Vertrauensfähigkeit wurde ebenfalls mittels eines vollständigen Messmodells erfasst. ${ }^{13}$ Abbildung 2 zeigt das Strukturgleichungsmodell zur Erklärung von Vertrauen in Themenselektivität.

Vertrauensfähigkeit hat hypothesenkonform einen signifikant positiven Einfluss $(\gamma=$ $.35 ; \mathrm{p}<.001)$ auf das Vertrauen in die Themenselektivität. Mit anderen Worten: Eine Person, die positiv in die Zukunft schaut, vertraut stärker darauf, dass sie alle relevanten und interessanten Themen durch den Journalismus erfährt, als eine Person, die der eigenen Zukunft eher negativ gegenüber steht. Das Vertrauen in die Themenselektivität wird zu $12 \%$ von der exogenen Variable Vertrauensfähigkeit erklärt. Diese hat ebenso einen signifikant positiven Effekt auf das Vertrauen in die Faktenselektivität $(\gamma=.31$; p <.001; Varianzerklärung: 9\%; GFI: .98 / AGFI: .95 / RMSEA: .06), auf die Glaubwürdigkeit $(\gamma=.37$; $<$.001; Varianzerklärung: 14\%; GFI: .97 / AGFI: .95 / RMSEA: .04) und auf das Vertrauen in journalistische Bewertungen $(\gamma=.34 ; \mathrm{p}<.001$; Varianzerklärung: 12\%; GFI: .99 / AGFI: .97 / RMSEA: .04). Aus Platzgründen wird auf eine graphische Darstellung dieser Strukturgleichungsmodelle verzichtet.

\section{Diskussion}

Das zentrale Ergebnis der Studie ist die vorläufige Bestätigung des postulierten Faktorenmodells zu Vertrauen in Journalismus. Wenn die befragten Personen ihr Vertrauen in Journalismus äußern, unterscheiden sie vier Faktoren: Vertrauen in die Themenselektivität, Vertrauen in die Faktenselektivität, Vertrauen in die Richtigkeit von Beschreibungen (Glaubwürdigkeit) und Vertrauen in Bewertungen. Überdies ergab die Analyse, dass sich der dritte Faktor, Richtigkeit von Beschreibungen resp. Glaubwürdigkeit, in die beiden Subdimensionen Vollständigkeit von Informationen und Korrektheit von Informationen aufsplittet. Interessant ist daran vor allem, dass die Vollständigkeit von Informationen als eine Variante der Richtigkeit von Beschreibungen zu

13 Die drei verwendeten Items (Verf1 bis Verf3) lauten: „Ich setze große Hoffnungen in die Zukunft.“ $(\lambda=.67) /$ „Das Leben wird mir noch viel mehr schöne Zeiten bringen als schlechte. “ $(\lambda=.77) /$ "Ich blicke mit Optimismus und Begeisterung in die Zukunft." $(\lambda=.83)$ Die Varianz von Vertrauensfähigkeit wurde auf 1 fixiert. 
begreifen wäre und nicht als Bestandteil der Faktenselektivität, also der angemessenen Kontextualisierung eines Themas. Es ist zu betonen, dass hiermit erstmalig die empirische Umsetzung eines mehrdimensionalen Modells von Vertrauen in Journalismus gelungen ist, das über den in bisherigen Untersuchungen fast ausschließlich fokussierten Aspekt der Glaubwürdigkeit „von Medien“ hinaus geht. Das Modell ermöglicht eine genauere Beschreibung von Vertrauen in Journalismus, da es sich zum Ersten an konkreten Erwartungen orientiert, die Rezipienten an den Journalismus richten, und zum Zweiten eine Differenzierung in vier Vertrauensfaktoren vornimmt. Allerdings muss einschränkend gesagt werden, dass sich die Antworten der Befragten auf die Berichterstattung zum Thema Euro beziehen. Man kann zwar davon ausgehen, dass die extrahierte Faktorenstruktur eine adäquate Beschreibung auch anderer Themen ermöglicht; dennoch muss dies in zukünftigen Untersuchungen erst empirisch gezeigt werden. Auch auf Grund des teilweise explorativen Vorgehens bei der konfirmatorischen Faktorenanalyse muss die Skala an einer neuen Stichprobe überprüft und validiert werden. Überdies ist die spezifische Formulierung und damit Inhaltsvalidität einzelner Items zu überdenken. Insbesondere wird sich hierbei zeigen, ob die Zuordnung der Subdimension Vollständigkeit von Informationen zur Richtigkeit von Informationen (dem Glaubwürdigkeitsfaktor) Bestand hat. Von Interesse sind zudem auch die hier noch nicht berücksichtigten Konstrukte Vertrautheit und Misstrauen (vgl. Luhmann 1989).

Ein zweites Ergebnis besteht in einer ersten Konstruktvalidierung der Skala zu Medienvertrauen. Es konnte gezeigt werden, dass die Vertrauensfähigkeit einer Person ein signifikanter Prädiktor aller Vertrauensfaktoren ist. Allerdings kann auch die Konstruktvalidierung mit diesem Ergebnis noch nicht als abgeschlossen gelten. In zukünftigen Untersuchungen sollte die Beziehung eines genuin konfirmatorischen Modells zu weiteren - bereits validierten - Konstrukten erfolgen. Überdies gilt zu fragen, welchen Stellenwert und welche Vorhersagekraft die vier Vertrauensfaktoren bei unterschiedlichen Themen und zu verschiedenen Zeitpunkten im Rezeptionsprozess haben.

Die Ergebnisse lassen daher weiterführende Untersuchungen sowohl notwendig als auch viel versprechend erscheinen: Liegt nämlich eine reliable und validierte Skala zur Erfassung von Vertrauen in Journalismus vor, besteht zum Ersten die Möglichkeit, das nun operationalisierte Vertrauenskonzept funktional in die Medienwirkungsforschung einzubinden. Beispielsweise könnte gefragt werden, welche Rolle Vertrauen in Journalismus für die Wirkung so genannter Medienframes hat. Vertrauen könnte somit als Schlüsselvariable bei der Prognose und Erklärung von spezifischen Medienwirkungen eingesetzt werden. Zum Zweiten würde ein solches Messinstrument auch einen informativeren Vergleich verschiedener redaktioneller Angebote, also z. B. zwischen Qualitätszeitungen und Regionalzeitungen, ermöglichen. Die Aufgliederung des Vertrauens in mehrere Faktoren erlaubte eine weitaus differenziertere Betrachtungsweise als bislang möglich (und üblich). Zum Dritten würde der Anwendungsbezug kommunikationsund medienwissenschaftlicher Forschung erweitert. Dies betrifft nicht nur die Diagnose von Vertrauensproblemen, sondern auch die "Therapie“ in Form konkreter Vorschläge für journalistisches Handeln.

\section{Literatur}

Al-Makaty, Safran S. / Boyd, Douglas A. / Tubergen, G. Norman van (1994): Source credibility during the Gulf war: a Q-study of rural and urban Saudi Arabian citizens. In: Journalism Quarterly, 71. Jg., S. $55-63$.

Barber, Bernard (1983): The logic and limits of trust. New Brunswick (NJ). 
Bentele, Günter (1988a): Objektivität und Glaubwürdigkeit von Medien. Eine theoretische und empirische Studie zum Verhältnis von Realität und Medienrealität. Unveröffentlichte Habilitationsschrift. Freie Universität Berlin, Fachbereich Kommunikationswissenschaften.

Bentele, Günter (1988b): Der Faktor Glaubwürdigkeit. Forschungsergebnisse und Fragen für die Sozialisationsperspektive. In: Publizistik, 33. Jg., S. 406 - 426.

Bentele, Günter (1994a): Objektivitätsanspruch und Glaubwürdigkeit. In: Jarren, Otfried (Hrsg.): Medien und Journalismus 1. Eine Einführung. Opladen, S. 295 - 312.

Bentele, Günter (1994b): Öffentliches Vertrauen - normative und soziale Grundlage für Public Relations. In: Armbrecht, Wolfgang / Zabel, Ulf (Hrsg.): Normative Aspekte der Public Relations. Opladen, S. $131-158$.

Bentele, Günter (1998): Vertrauen / Glaubwürdigkeit. In: Jarren, Otfried / Sarcinelli, Ulrich / Saxer, Ulrich (Hrsg.): Politische Kommunikation in der demokratischen Gesellschaft. Ein Handbuch mit Lexikonteil. Opladen, S. $305-311$.

Berg, Klaus / Kiefer, Marie-Luise (Hrsg.) (1996): Massenkommunikation V. Eine Langzeitstudie zur Mediennutzung und Medienbewertung 1964 - 1995. Baden-Baden.

Bollen, Kenneth A. (1989): Structural equations with latent variables. New York.

Bryant, Fred B. (2000): Assessing the validity of measurement. In: Grimm, Laurence G. / Yarnold, Paul R. (Hrsg.): Reading and understanding more multivariate statistics. Washington, DC, S. $99-146$.

Byrne, Barbara M. (2001): Structural equation modeling with AMOS. Basic concepts, applications, and programming. Mahwah (N.J.)/London.

Chanley, Virgina A. / Rudolph, Thomas J. / Rahn, Wendy M. (2000): The origins and consequences of public trust in government. A time series analysis. In: Public Opinion Quarterly, 64. Jg., S. $239-256$.

Coleman, James S. (21994): Foundations of social theory. Cambridge (MA) / London.

Cronkhite, Gary / Liska, Jo (1976): A critique of factor analytic approaches to the study of credibility. In: Speech Monographs, 43. Jg., S. $91-107$.

Drescher, Siegfried H. (1969): Wirkung des Fernsehens als Quelle aktueller Informationen. In: Publizistik, 14. Jg., S. $173-184$.

Earle, Timothy C. / Cvetkovich, George T. (1995): Social trust. Toward a cosmopolitan society. Westport (Connecticut) / London.

Edelstein, Alex S. (1978): An alternative approach to the study of source effects in MASS communication. In: Communications, 4. Jg., S. 71 - 90.

Gantz, Walter (1981): The influence of researcher methods on television and newspaper news credibility evaluations. In: Journal of Broadcasting, 25. Jg., S. $155-169$.

Gaziano, Cecilie (1988): How credible is the credibility crisis? In: Journalism Quarterly, 65. Jg., S. $267-278,375$.

Gaziano, Cecilie / McGrath, Kristin (1986): Measuring the concept of credibility. In: Journalism Quarterly, 63. Jg., S. $451-462$.

Gerbing, David W. / Hamilton, Janet G. (1996): Viability of exploratory factor analysis as a precursor to confirmatory factor analysis. In: Structural Equation Modeling, 3. Jg., Nr. 1, S. 62 72.

Giddens, Anthony (21997): Konsequenzen der Moderne (Orig. 1990). Frankfurt am Main.

Gorsuch, Richard L. (1983): Factor analysis. Hillsdale (NJ).

Greenberg, Bradley S. / Razinsky, Edward L. (1966): Some effects of variations in message quality. In: Journalism Quarterly, 43. Jg., S. $486-492$.

Hardin, Russell (2002): Trust and trustworthiness. New York.

Hartmann, Martin / Offe, Claus (Hrsg.) (2001): Vertrauen. Die Grundlage des sozialen Zusammenhalts. Frankfurt am Main.

Homburg, Christian / Giering, Annette (1998): Konzeptualisierung und Operationalisierung komplexer Konstrukte - Ein Leitfaden für die Marketingforschung. In: Hildebrandt, Lutz / Homburg, Christian (Hrsg.): Die Kausalanalyse. Ein Instrument der betriebswirtschaftlichen empirischen Forschung. Stuttgart, S. $111-146$.

Homburg, Christian / Pflesser, Christian (2000): Konfirmatorische Faktorenanalyse. In: Herr- 
mann, Andreas / Homburg, Christian (Hrsg.): Marktforschung. Methoden - Anwendungen Praxisbeispiele. 2., aktualisierte Auflage. Wiesbaden, S. 413 - 437.

Hoyle, Rick / Panter, Abigail T. (1995): Writing about structural equation models. In: Hoyle, Rick (Hrsg.): Structural equation modelling. Thousand Oaks (CA), S. $158-176$.

Hoyle, Rick (2000): Confirmatory factor analysis. In: Tinsley, Howard E. A. / Brown, Steven D. (Hrsg.): Handbook of applied multivariate statistics and mathematical modeling. San Diego, S. $465-497$.

Hovland, Carl I. / Janis, Irving L. / Kelley, Harold H. ( $\left.{ }^{3} 1959\right)$ : Communication and persuasion. Psychological studies of opinion change (1953). New Haven.

$\mathrm{Hu}$, Li-tze / Bentler, Peter M. (1999): Cutoff criteria for fit indexes in covariance structure analysis: conventional criteria versus new alternatives. In: Structural Equation Modeling, 6. Jg., S. $1-55$.

Jacobson, Harvey K. (1969): Mass media believability: a study of receiver judgements. In: Journalism Quarterly, 46. Jg., S. 20 - 28.

John, Oliver P. / Benet-Martínez, Veronica (2000): Measurement: reliability, construct validation, and scale construction. In: Reis, Harry T. / Judd, Charles M. (Hrsg.): Handbook of research methods in personality and social psychology. New York, S. 339-369.

Johnson, Thomas J. / Kaye, Barbara K. (1998): Cruising is believing? Comparing Internet and traditional sources on media credibility measures. In: Journalism \& Mass Communication Quarterly, 75. Jg., S. 325 - 340.

Kelle, Uwe/ Erzberger, Christian (1999): Integration qualitativer und quantitativer Methoden. Methodologische Modelle und ihre Bedeutung für die Forschungspraxis. In: Kölner Zeitschrift für Soziologie und Sozialpsychologie, 51. Jg., S. 509-531.

Kline, Rex B. (1998): Principles and practice of structural equation modeling. London.

Kohring, Matthias (1997): Die Funktion des Wissenschaftsjournalismus. Ein systemtheoretischer Entwurf. Opladen.

Kohring, Matthias (2000): Komplexität ernst nehmen. Grundlagen systemtheoretischer Journalismustheorie. In: Löffelholz, Martin (Hrsg.): Theorien des Journalismus. Opladen, S. $153-168$.

Kohring, Matthias (2001): Vertrauen in Medien - Vertrauen in Technologie. Stuttgart.

Kohring, Matthias (2002a): Vertrauen in Journalismus. In: Scholl, Armin (Hrsg.): Systemtheorie und Konstruktivismus in der Kommunikationswissenschaft: Ansprüche und Herausforderungen. Konstanz, S. $91-110$.

Kohring, Matthias (2002b): Fakten ins Töpfchen, Fiktionen ins Kröpfchen? Warum Vertrauen in Journalismus mehr ist als Glaubwürdigkeit. In: Baum, Achim / Schmidt, Siegfried J. (Hrsg.): Fakten und Fiktionen: Über den Umgang mit Medienwirklichkeiten. Konstanz, S. $90-100$.

Krampen, Günther (1994): Skalen zur Erfassung von Hoffnungslosigkeit. Göttingen.

Krampen, Günther (1997): Zur handlungs-, persönlichkeits- und entwicklungspsychologischen Einordnung des Konstrukts Vertrauen. In: Schweer, Martin K. W. (Hrsg.): Vertrauen und soziales Handeln. Facetten eines alltäglichen Phänomens. Neuwied, S. 16-61.

Lee, Raymond S. H. (1978): Credibility of newspaper and TV news. In: Journalism Quarterly, 55. Jg., S. $282-287$.

Luhmann, Niklas (1989): Vertrauen. Ein Mechanismus der Reduktion sozialer Komplexität. 3., durchgesehene Auflage (1968). Stuttgart.

Matthes, Jörg (2001): Operationalisierung von Vertrauen in Medien. Zur empirischen Erklärung einer riskanten Handlung. Unveröffentlichte Diplomarbeit. Friedrich-Schiller-Universität Jena, Institut für Psychologie.

McCroskey, James C. / Young, Thomas J. (1979): The use and abuse of factor analysis in communication research. In: Human Communication Research, 4. Jg., S. 375 - 382.

McCroskey, James C. / Young, Thomas, J. (1981): Ethos and credibility: The construct and its measurement after three decades. In: Central States Speech Journal, 32. Jg., S. $24-34$.

Meyer, Timothy (1974): Media credibility: the state of the research. In: Public Telecommunications Review, 2. Jg., Nr. 4, S. 48-52.

Misztal, Barbara (1996): Trust in modern societies. The search for the bases of social order. Cambridge. 
Mulder, Ronald (1980): Media credibility: a use-gratifications approach. In: Journalism Quarterly, 57. Jg., S. $474-477$.

Nawratil, Ute (1997): Glaubwürdigkeit in der sozialen Kommunikation. Opladen.

Newhagen, John / Nass, Clifford (1989): Differential criteria for evaluating credibility of newspapers and TV news. In: Journalism Quarterly, 66. Jg., S. 277 - 284.

Robinson, Michael J. / Kohut, Andrew (1988): Believability and the press. In: Public Opinion Quarterly, 52. Jg., S. $174-189$.

Rindskopf, David / Rose, Tedd (1988): Some theory and application of confirmatory second-order factor analysis. In: Multivariate Behavioral Research, 23. Jg., S. 51 - 67.

Roper, Burns W. (1985): Public attitudes toward television and other media in a time of change. New York.

Schumaker, Randall E. / Lomax, Richard G. (1996): A beginner's guide to structural equation modeling. Mahwah (NJ).

Self, Charles C. (1996): Credibility. In: Salwen, Michael B. / Stacks, Don. W. (Hrsg.): an integrated approach to communication theory and research. Mahwah, NJ, S. $421-444$.

Shaw, Eugene F. (1973): Media credibility: taking the measure of a measure. In: Journalism Quarterly, 50. Jg., S. $306-311$.

Simmel, Georg ( $\left.{ }^{3} 1999\right)$ : Soziologie. Untersuchungen über die Formen der Vergesellschaftung. Hrsg. von Otthein Rammstedt. Band 11 der Gesamtausgabe (Orig. 1908).

Sztompka, Piotr (1999): Trust. A sociological theory. Cambridge.

Tashakkori, Abbas / Teddlie, Charles (1998): Mixed methodology - combining qualitative and quantitative approaches. London.

Wanta, Wayne/ Hu, Yu-Wei (1994): The effects of credibility, reliance, and exposure on media agenda-setting: a path analysis model. In: Journalism Quarterly, 71. Jg., S. 90 - 98.

West, Mark D. (1994): Validating a scale for the measurement of credibility: a covariance structure modeling approach. In: Journalism Quarterly, 71. Jg., S. 159 - 168.

Westley, Bruce / Severin, Werner (1964): Some correlates of media credibility. In: Journalism Quarterly, 41. Jg., S. $325-335$.

Wirth, Werner (1999): Methodologische und konzeptionelle Aspekte der Glaubwürdigkeitsforschung. In: Rössler, Patrick / Wirth, Werner (Hrsg.): Glaubwürdigkeit im Internet. Fragestellungen, Modelle, empirische Befunde. München, S. 47 - 66. 